Instituto Internacional de Investigación y Desarrollo Tecnológico Educativo INDTEC, C.A.

DOI: https://doi.org/10.29394/Scientific.issn.2542-2987.2020.5.15.10.209-228

OAI-PMH: http://www.indteca.com/ojs/index.php/Revista Scientific/oai

Artículo Original / Original Article

\title{
Proyectos comunitarios: Una experiencia didáctica en Formación Comunitaria
}

Autora: Lisbeth María Cedeño de Veracierto

\section{Resumen}

El presente artículo, tiene como propósito exponer los resultados de una Investigación Acción Participativa cuyo objetivo consistió en desarrollar un Programa de Formación Comunitaria mediante el cual los participantes aprendieran a realizar Proyectos Comunitarios. Dirigido a integrantes del Consejo Comunal del Barrio Los Naranjos en el Municipio Valencia, estado Carabobo, Venezuela. Sustentado en la Teoría de las Necesidades de Maslow. Para su ejecución se aplicó un instrumento dicotómico a fin de diagnosticar las necesidades y la aplicabilidad del programa, estructurado de la siguiente manera: Una I Parte, exploratoria en cuanto al conocimiento previo de proyectos comunitarios. Una II Parte, para precisar el interés de participación de los consultados en desarrollar proyectos comunitarios. Y una III Parte, diseñada para determinar la necesidad de implementar un programa de formación comunitaria. De los resultados obtenidos del diagnóstico se determinó la necesidad de crear un programa formativo en función de satisfacer la necesidad cognitiva que les facilitara realizar sus propios proyectos comunitarios. Finalmente se concluyó que mediante la participación comunitaria en gestión de proyectos estas pueden empoderarse y autogestionar su desarrollo social al ser parte en la toma de decisiones relacionadas a políticas públicas que les competan.

Palabras clave: formación; participación comunitaria; desarrollo social.

Cómo citar este artículo:

Cedeño de Veracierto, L. (2020). Proyectos comunitarios: Una experiencia didáctica en Formación Comunitaria. Revista Scientific, 5(15), 209-228, e-ISSN: 2542-2987. Recuperado de: https://doi.org/10.29394/Scientific.issn.2542-2987.2020.5.15.10.209-228

Fecha de Recepción:

10-07-2019
Fecha de Aceptación:

05-10-2019
Fecha de Publicación:

05-02-2020 


\title{
Community projects: A didactic experience in Community Formation
}

\begin{abstract}
The purpose of this article is to present the results of a Participatory Action Research whose objective was to develop a Community Training Program through which participants learned to carry out Community Projects. Aimed at members of the Los Naranjos Neighborhood Community Council in the Municipality of Valencia, Carabobo state, Venezuela. Based on Maslow's Theory of Needs. For its execution, a dichotomous instrument was applied in order to diagnose the needs and applicability of the program, structured as follows: An I Part, exploratory regarding prior knowledge of community projects. A Part II, to specify the interest of participation of those consulted in developing community projects. And a Part III, designed to determine the need to implement a community formation program. From the results obtained from the diagnosis, the need to create a training program based on satisfying the cognitive need that facilitated them to carry out their own community projects was determined. Finally, it was concluded that through community participation in project management, they can be empowered and self-manage their social development by being part of the decision-making related to public policies that compete with them.
\end{abstract}

Keywords: training; community participation; social development.

How to cite this article:

Cedeño de Veracierto, L. (2020). Community projects: A didactic experience in Community Formation. Revista Scientific, 5(15), 209-228, e-ISSN: 2542-2987. Recovered from: https://doi.org/10.29394/Scientific.issn.2542-2987.2020.5.15.10.209-228

Date Received:

10-07-2019
Date Acceptance:

05-10-2019
Date Publication:

05-02-2020 


\section{Introducción}

La dinámica en la que se desarrollan las sociedades en la actualidad, requiere de comunidades organizadas dispuestas a la adaptación de constantes transformaciones, que van desde la estructura organizativa hasta los modelos de desarrollo social definidos por las políticas públicas de los entes gubernamentales donde se encuentran. En este sentido la educación comunitaria cobra mayor fuerza debido a la necesidad de formar a las comunidades a convivir y desarrollarse a la par de los cambios a los que deban someterse. Siendo los programas de formación comunitaria el medio a través del cual estas comunidades encuentran el apoyo para el desarrollo de proyectos propicios a sus necesidades.

En consonancia con lo anterior, el presente artículo tiene como propósito compartir los resultados de una experiencia didáctica generada de la aplicación de una Investigación Acción participativa, cuyo objetivo consistió en ejecutar un programa de formación en proyectos comunitarios que permitiera a los miembros de la Comunidad del Barrio Los Naranjos, ubicada en el Municipio Valencia del Estado Carabobo en Venezuela, aprender a realizar proyectos comunitarios. En este orden de ideas, es pertinente saber que, para el desarrollo del referido trabajo de investigación, se diseñó y aplicó un cuestionario dicotómico con el fin de medir la viabilidad de desarrollar un programa de formación comunitaria.

En función de los resultados obtenidos se determinó su viabilidad, siendo aplicada en la comunidad antes citada. A tales efectos, cabe destacar que para el desarrollo de la investigación se procedió inicialmente al diagnóstico de las necesidades de la comunidad en cuestión. Mediante el cual se pudo determinar cuáles eran las necesidades más prioritarias dentro del plano de la organización comunitaria que requería mayor asistencia. Por lo que se observó como principal necesidad realizar proyectos comunitarios a través de los cuales pudieran solucionar gran parte de sus necesidades colectivas. Luego 
de ello los resultados obtenidos sirvieron de referencia para la creación de un plan de acción que condujo al diseño de un programa de formación para la elaboración de proyectos comunitarios dirigido a los responsables del Consejo Comunal de la comunidad antes citada.

\section{Percepción del investigador}

Las organizaciones comunitarias del Siglo XXI desarrolladas en contextos socioculturales como los de Latinoamérica, demuestran cada vez mayor conciencia de sus necesidades de participación ciudadana y capacidad para la autogestión de sus comunidades. Igualmente, estas parecen comprender que la dinámica de los últimos tiempos demanda una ciudadanía menos dependiente del Estado y más empoderadas en la toma de decisiones para la solución de los diversos problemas comunitarios.

Igualmente es comprensible que existen determinadas situaciones en las cuales se impone la intervención del Estado cuando de problemas complejos se trata. En este sentido, los proyectos comunitarios se han constituido en la vía más propicia mediante la cual las comunidades organizadas pueden canalizar sus necesidades. En este mismo tenor, la Ley Orgánica de los Consejos Comunales (2010a), en su Artículo 4, numeral 7, lo define como: “...el conjunto de actividades concretas orientadas a lograr uno o varios objetivos, para dar respuesta a las necesidades, aspiraciones y potencialidades de las comunidades" (pág. 15).

Desde esta perspectiva es oportuno considerar que la participación comunitaria reclama más que nunca acción y protagonismo en sus procesos de formación para la gestión comunitaria. Al respecto, Requena (2018), refiere que: "la participación comunitaria es un proceso cambiante y variable, lo cual está vinculado con las actitudes, toma de decisiones y relación entre los individuos" (pág. 295); por lo que se infiere que la participación comunitaria adquiere relevancia en el contexto de las comunidades organizadas. 
En Venezuela, las comunidades organizadas cuentan con una estructura social conocida como Consejo Comunal, cuya organización social hoy por hoy constituye la figura legal sobre la cual las comunidades fundamentan y median su participación comunitaria. Estos Consejos Comunales están definidos según la Ley Orgánica de los Consejos Comunales (2010b), como:

(...) instancias de participación, articulación e integración ente los ciudadanos, ciudadanas y las diversas organizaciones comunitarias, movimientos sociales y populares, que permiten al pueblo organizado ejercer el gobierno comunitario y la gestión directa de políticas públicas y proyectos orientados a responder a las necesidades, potencialidades y aspiraciones de las comunidades, en la construcción del nuevo modelo de sociedad socialista de igualdad, equidad y justicia social (pág. 13).

No obstante, si bien es cierto que las comunidades organizadas cuentan con esta base legal que promueve la gestión directa de políticas públicas y el impulso de realizar proyectos comunitarios, también es cierto que en ocasiones, algunas comunidades requieren de asesoría para el desarrollo de su rol protagónico dentro de la estructura social de participación ciudadana.

\section{Referentes teóricos}

\subsection{Teoría de las necesidades de Abraham Maslow}

En relación al tema de las necesidades, para Durkheim (1982), citado por Mballa (2017): "la sociedad cuenta entre sus atributos con el de poder definir sus propias necesidades..." (pág. 104). En este mismo orden de ideas, es preciso hacer referencia a la Teoría de las Necesidades de Abraham Maslow en la cual plantea una jerarquización de las necesidades humanas, estructuradas en forma piramidal, ubicando en la base las necesidades inferiores y sobre estas las necesidades superiores, calificadas así por el autor. Estas según Maslow (1991): "tienen propiedades diferentes, pero son iguales en que tanto las unas como las otras deben incluirse en el repertorio de la 
naturaleza humana" (pág. 87).

Por su parte, Palomo (2010): aclara que las necesidades inferiores a las que se refiere Maslow tienen que ver con las necesidades fisiológicas y de seguridad y las necesidades superiores con afecto, autoestima y autorrealización (pág. 94). En tal sentido, Maslow también plantea que en la medida que se satisfagan razonablemente estas necesidades partiendo desde la base piramidal que el propone, entonces se activaran las necesidades consecutivas en orden ascendente. Ya que por naturaleza el hombre procura estar protegido contra el peligro o la privación, por lo que requiere sentir seguridad y vivir en un ambiente agradable, en mantenimiento del orden para él y para su familia.

\section{Metodología}

Se trató de una investigación de campo, enmarcada en lo que se conoce como Investigación Acción-Participativa. En relación a la definición de investigación de campo, según el Manual de Trabajos de Grado de Especialización y Maestría y Tesis Doctorales de la Universidad Pedagógica Experimental Libertador (UPEL, 2016):

Se entiende por Investigación de Campo, el análisis sistemático de problemas en la realidad, con el propósito bien sea de describirlos, interpretarlos, entender su naturaleza y factores constituyentes, explicar sus causas y efectos, o predecir su ocurrencia, haciendo uso de métodos característicos de cualquiera de los paradigmas o enfoques de investigación conocidos o en desarrollo (...) (pág. 18).

En este sentido, la investigación sobre la cual este artículo se fundamenta estuvo desarrollada en una comunidad organizada anteriormente descrita, de la cual se obtuvo el conjunto de datos necesarios para la ejecución y el análisis posterior de los resultados. En cuanto a la Investigación Acción Participativa, Eizagirre y Zabala (2006): se refieren a esta, como un "método 
de investigación y aprendizaje colectivo de la realidad, basado en un análisis crítico con la participación activa de los grupos implicados, que se orienta a estimular la práctica transformadora y el cambio social" (párr. 1); lo cual es útil, para el constructo de este tipo de investigación.

Igualmente, López de Ceballos (2008), citado por Páez (2017), destaca que en este método: "la participación no es exclusiva de los investigadores profesionales, sino que el papel protagónico lo tiene la comunidad en la cual se desarrolla la acción" (pág. 15). Por tal motivo se estructuró de la siguiente manera; partiendo de una primera fase donde se buscó diagnosticar las necesidades sobre las cuales estuvo basada la investigación, seguido de una segunda fase en la que se planteó una propuesta y una tercera fase de desarrollo de la misma, concluyendo con la sistematización de la experiencia.

\section{Resultados y análisis de la investigación}

\subsection{Fase: El Diagnóstico}

Para el desarrollo de esta investigación se aplicó un cuestionario dirigido a treinta miembros del Consejo Comunal de la Comunidad Los Naranjos, estructurado de la siguiente manera: Una I Parte, en búsqueda de explorar el conocimiento previo en cuanto a proyectos comunitarios; una II Parte, cuyo objetivo fue describir el nivel de participación de los miembros de la comunidad para desarrollar proyectos comunitarios; y una III Parte, diseñada para determinar la necesidad de implementar un programa de formación comunitaria en diseño y ejecución de proyectos en la comunidad del Barrio Los Naranjos. En el gráfico 1, se muestran los resultados obtenidos del instrumento aplicado, considerando una selección de tres preguntas determinantes para el diagnóstico de la investigación. 
Gráfico 1. Resultados y análisis.

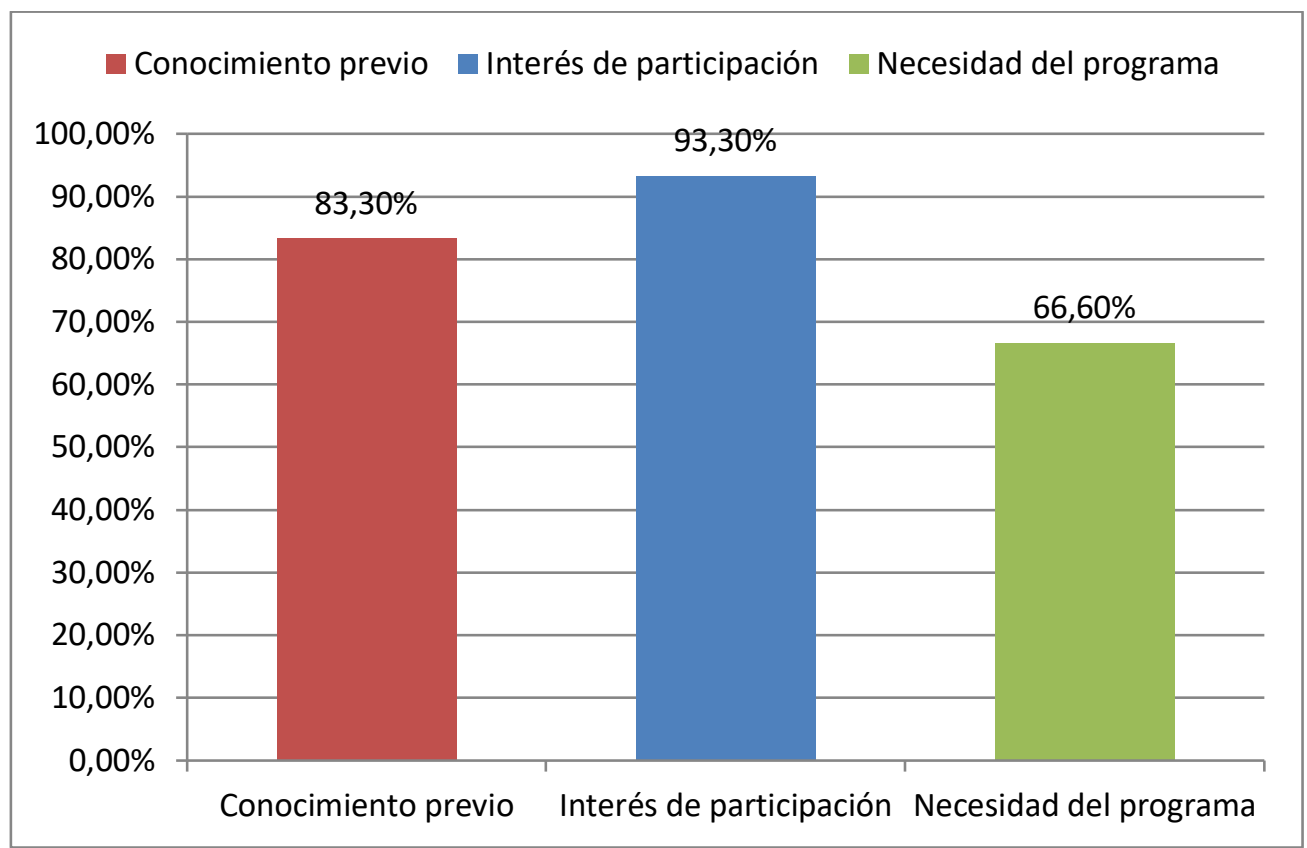

Fuente: La Autora (2019).

En la primera Parte del cuestionario, cuya variable estuvo enfocada en precisar el conocimiento previo sobre los proyectos comunitarios, tal como se visualiza en el gráfico 1 , donde se refleja los resultados del cuestionario aplicado. Puede observarse claramente que un $83,30 \%$ respondió no saber cómo realizar un proyecto comunitario, mientras que por el contrario el 16,6\% restante manifestó saber cómo hacerlo. Lo que sirvió de referencia para considerar el hecho de desarrollar un programa de formación comunitaria dirigido a quienes manifestaron desconocer sobre el tema.

En cuanto a la segunda Parte, es visible cómo en la variable relativa al interés en participar en un Taller de Proyectos Comunitarios, igualmente representado en el gráfico 1, un 93,30\% respondió afirmativamente, en tanto que otro $6,6 \%$ se limitó a responder que no, ante la misma pregunta sobre si participaría en un Taller de Formación en Proyectos Comunitarios.

A propósito de la Tercera parte del cuestionario, igualmente observable 
en el gráfico 1, esta se enfocó en determinar la necesidad de desarrollar el Programa de Formación Comunitaria. En torno a lo cual se les preguntó a las personas ¿Cree Usted que los Proyectos Comunitarios tengan algún beneficio para la comunidad?. Ante lo cual por una parte un $66,60 \%$ respondió que sí y por otra parte un $33,30 \%$ respondió que no. En función de lo cual se consideró la existencia de una necesidad que justificaría desarrollar el Programa en cuestión.

A fin de ilustrar los resultados obtenidos para el desarrollo del trabajo de investigación aplicado, se ha sintetizado la información del diagnóstico en la siguiente tabla 1.

Tabla 1. Resultados de la aplicación del cuestionario dicotómico.

\begin{tabular}{|c|c|}
\hline Parte I & $\begin{array}{l}\text { Variables: Conocimiento previo sobre los Proyectos Comunitarios. } \\
\text { Preguntas: } N^{\circ} 6 \text { ¿Conoce usted cómo elaborar un Proyecto Comunitario? } \\
\text { Respuestas: } 83,30 \% \text { Expresó que no sabía cómo realizar un Proyecto } \\
\text { Comunitario, el } 16,60 \% \text { dijo que sí. } \\
\text { Conclusión: Aunque un } 16,60 \% \text { de las personas admitió saber de qué se } \\
\text { trataba el tema consultado también manifestaron no tener interés en aprender } \\
\text { sobre el mismo, en tanto que un } 83,30 \% \text { igualmente consultado expresó estar } \\
\text { de acuerdo en aprender. }\end{array}$ \\
\hline Parte II & $\begin{array}{l}\text { Variables: Interés en la participación del Taller en Proyectos Comunitarios. } \\
\text { Preguntas: En la pregunta Nº ¿Participaría en un Taller de Formación en } \\
\text { Proyectos Comunitarios? } \\
\text { Respuestas: Un } 93,30 \% \text { manifestó estar de acuerdo mientras que } 6,60 \% \\
\text { respondió que no. } \\
\text { Conclusión: Se pudo observar en este resultado en particular una importante } \\
\text { demanda que sirvió de referencia al momento de considerar el diseño de un } \\
\text { Taller en Proyectos comunitarios. }\end{array}$ \\
\hline Parte II & $\begin{array}{l}\text { Variables: Necesidad del Programa de Formación Comunitaria. } \\
\text { Preguntas: } N^{\circ} 17 \text { ¿Cree usted que los Proyectos Comunitarios tengan algún } \\
\text { beneficio para su comunidad? } \\
\text { Respuestas: El } 66,60 \% \text { de los consultados respondió que sí, mientras que } \\
\text { un } 33,30 \% \text { expresó que no. } \\
\text { Conclusión: Considerando la magnitud de la respuesta afirmativa en } \\
\text { contraste con la negativa se determinó la necesidad de implementar un Taller } \\
\text { para la construcción de Proyectos Comunitarios como parte del Programa de } \\
\text { Formación Comunitaria. }\end{array}$ \\
\hline
\end{tabular}

Fuente: La Autora (2019).

De los resultados obtenidos del diagnóstico que se detallan más adelante, se determinó la necesidad de crear una propuesta en función de 
satisfacer la necesidad cognitiva que les facilitara realizar sus propios proyectos comunitarios. Ante lo cual surge la propuesta de un Programa de Formación Comunitaria dirigido a miembros de la Comunidad Los Naranjos.

Tal como se observó en la tabla 1, en la cual se reflejan los resultados de las preguntas dicotómicas aplicadas a los miembros del Consejo Comunal del Barrio Los Naranjos.

\subsection{Fase: La Propuesta}

Asimismo, es pertinente destacar que la educación es un proceso sistematizado del conocimiento estrechamente asociado a los procesos de formación educativa, cuyo valor incalculable la convierte en un derecho según la Ley Orgánica de Educación (2009a), en el artículo 14, el cual cita lo siguiente en su texto preliminar: "la educación es un derecho humano y un deber social fundamental concebida como un proceso de formación integral, gratuita, laica, inclusiva y de calidad, permanente, contínua e interactiva..." (pág. 8).

Igualmente, en el numeral 2 del artículo 15, de esta misma Ley Orgánica de Educación (2009b), refiere como uno de los fines de la educación:

Desarrollar una nueva cultura política fundamentada en la participación protagónica y el fortalecimiento del Poder Popular, en la democratización del saber y en la promoción de la escuela como espacio de formación de ciudadanía y de participación comunitaria, para la reconstrucción del espíritu público en los nuevos republicanos y en las nuevas republicanas con profunda conciencia del deber social (págs. 8-9).

De igual forma, y en cuanto a la importancia de la formación educativa, la Ley Orgánica de los Consejos Comunales (2010c): en su artículo 25, titulado Funciones del Colectivo de Coordinación Comunitaria, señala en el numeral 10, que una de las funciones de estos, es la de "promover la formación y capacitación comunitaria en los voceros y voceras del Consejo Comunal y en 
la comunidad en general" (pág. 25).

Sobre las bases antes expuesta, se procedió a diseñar como propuesta un Programa de Formación Comunitaria. Estructurado por tres encuentros formativos. El primero de ellos consistió en un Foro cuyo tema estuvo centrado en el contexto legal de la gestión comunitaria y el rol del Estado como garante en los procesos de formación comunitaria, el segundo se trató de un taller práctico de cómo realizar proyectos comunitarios y un tercer encuentro donde los participantes expusieron sus propuestas como producto de lo aprendido durante los dos primeros encuentros.

Por su parte los participantes de este programa contarían además con la orientación de expertos durante el proceso de formación. Dada su relación al trabajo comunitario, Brizuela, Sáez y Jústiz (2015): consideran que, para poder cumplir con sus expectativas educativas, este tipo de proyecto debe "lograr la activa participación de la población en la transformación de su entorno" (pág. 112). En consonancia con lo anterior, el Programa de Formación Comunitaria, procuró en esta fase del proyecto acercar a la comunidad, a las instituciones del Estado que se dedican al proceso continuo de formación comunitaria, a fin de crear lazos de interés común entre todas las partes involucradas y comprometidas por una transformación comunitaria.

En función de lo anteriormente expuesto, es pertinente señalar -tal como se observa en la tabla 2, en cuanto al Plan de Acción diseñado para el desarrollo del Programa- que el mismo estuvo estructurado en tres sesiones o encuentros. Siendo el primero destinado a comprender el marco jurídico y el rol del Estado en a formación comunitaria. Para lo cual se planificó realizar un foro con la participación de especialistas y la intervención oportuna de los miembros del Consejo Comunal Los Naranjos. Igualmente se dispuso un segundo encuentro en el cual se planificó desarrollar un taller práctico donde los miembros del Consejo Comunal tuviesen la oportunidad de aprender a realizar proyectos comunitarios. Y un tercer encuentro donde los participantes 
del taller expondrían sus propuestas de proyectos comunitarios.

Tabla 2. Plan de Acción.

\begin{tabular}{|c|c|}
\hline Acció & \\
\hline $\begin{array}{l}\text { I Encuentro. } \\
\text { Marco teórico y jurídico. } \\
\text { (4 horas) }\end{array}$ & $\begin{array}{l}\text { Objetivos específicos: Comprender el marco jurídico y } \\
\text { el rol del Estado en la formación comunitaria. } \\
\text { Estrategia: Foro con panel de especialistas invitados. } \\
\text { Recursos: Video Beam, computadora, sala con sillas, } \\
\text { humanos. } \\
\text { Participantes: Miembros del consejo comunal Los } \\
\text { Naranjos, invitados. }\end{array}$ \\
\hline $\begin{array}{l}\text { II Encuentro. } \\
\text { Taller práctico: Cómo elaborar } \\
\text { proyectos comunitarios. } \\
\text { (4 horas) }\end{array}$ & $\begin{array}{l}\text { Objetivos específicos: Aprender a realizar proyectos } \\
\text { comunitarios. } \\
\text { Estrategia: Taller práctico realizado por un especialista. } \\
\text { Recursos: Video Beam, computadora, sala con sillas, } \\
\text { humanos. } \\
\text { Participantes: Miembros del consejo comunal Los } \\
\text { Naranjos. Facilitador. }\end{array}$ \\
\hline $\begin{array}{l}\text { III Encuentro. } \\
\text { Exposición de proyectos. } \\
\text { (2 horas) }\end{array}$ & $\begin{array}{l}\text { Objetivos específicos: Evaluar el aprendizaje adquirido } \\
\text { en los dos primeros encuentros. } \\
\text { Estrategia: Exposición. } \\
\text { Recursos: Video Beam, computadora, sala con sillas, } \\
\text { humanos. } \\
\text { Participantes: Miembros del consejo comunal Los } \\
\text { Naranjos. Facilitador. }\end{array}$ \\
\hline
\end{tabular}

Fuente: La Autora (2019).

\subsection{Fase: Desarrollo del Programa de Formación Comunitaria}

Durante la etapa de la aplicación del programa, se desarrollaron los siguientes encuentros (I Encuentro, II Encuentro, III Encuentro), tal y como se proyectó en el plan de acción representado en la tabla 2.

\subsubsection{Encuentro}

Foro: El rol formativo del Estado y la participación ciudadana de los Consejos Comunales.

Objetivo General: Motivar a los miembros de la Comunidad del Barrio Los Naranjos a desarrollar proyectos comunitarios sostenibles y productivos.

\section{Objetivos Específicos:}

- Comprender el contexto jurídico del Estado y su rol formativo respecto 
a la participación ciudadana de las comunidades.

- Conocer los programas e instituciones del Estado de formación comunitaria que puedan orientar a la comunidad a ejecutar proyectos sostenibles y productivos.

- Articular acciones entre la comunidad y las instituciones del Estado.

Desarrollo del Foro: El taller estuvo estructurado en dos sesiones de 90 (noventa) minutos cada una, con un receso entre ambas ponencias de 15 (quince) minutos. La primera ponencia fue ofrecida por una representante de la Oficina de Asistencia Técnica y Capacitación al Sector Productivo de la Corporación para el Desarrollo de la Región Central (CORPOCENTRO). Cuya ponencia estuvo centrada en el tema del foro, destacando el marco jurídico e institucional bajo el cual el Estado se compromete con la ciudadanía en apoyar las iniciativas de desarrollo bajo una orientación técnica que posibilite el logro de las mismas, a través de programas de formación comunitaria como los emprendidos por CORPOCENTRO desde que se inició el proceso de cambios que legitima la participación ciudadana de las comunidades.

Posterior al receso, se llevó a cabo la intervención de dos representantes del Departamento de Formación Profesional del Instituto Nacional de Capacitación y Educación Socialista (INCES). En tal sentido, los ponentes antes referidos enfocaron su presentación en los programas de capacitación que el INCES ofrece a las comunidades organizadas. Dando como ejemplo a una comunidad ubicada en el Barrio 13 de Septiembre, en el municipio Valencia, donde la comunidad se organizó para dar solución a un problema de electricidad que tenía una escuela de esa localidad. De tal forma que el INCES a través de uno de sus programas de formación y capacitación, instruyó a un grupo a la vez que les propiciaron los recursos técnicos y materiales para que la misma comunidad realizara la instalación eléctrica 
averiada.

\subsubsection{Encuentro}

Taller: Diseño y evaluación de Proyectos Comunitarios. ¿Cómo realizar Proyectos Comunitarios?

Objetivo General: Aprender a realizar Proyectos Comunitarios sostenibles en el tiempo.

\section{Objetivos Específicos:}

- Conocer en qué consiste un proyecto comunitario.

- Analizar la complejidad y prioridad de las necesidades que afectan a la comunidad.

- Valorar la planificación de proyectos como alternativa para la solución de problemas comunes.

Desarrollo del Taller: El Taller de ¿Cómo elaborar Proyectos Comunitarios sostenibles en el tiempo?, se efectuó en la Escuela Básica Dr. Francisco Espejo, ubicada en la Av. Principal del Barrio Monumental de la Parroquia Miguel Peña del Municipio Valencia, Edo. Carabobo. El mismo estuvo bajo la responsabilidad y conducción de la Profesora Lisbeth Cedeño, responsable de este proyecto y, quien procedió a dictar el taller haciendo una presentación bastante didáctica y adaptada al público que asistió. Comenzando por definir el concepto de Proyecto y continuando con puntos que condujera a los presentes a una mejor comprensión del tema. Luego de las definiciones y una ligera metacognición en la que los participantes manifestaron la comprensión de lo tratado, los participantes contaron con un breve receso de quince minutos para luego continuar con el taller. Después del receso, se procedió al desarrollo de las mesas de trabajo, colocando a los participantes en grupos de tres y cuatro participantes, ubicándolos por calles 
afines de sus residencias.

Se realizó un sorteo con notas en los que estaba escrito un problema comunitario. Cada grupo trabajó en función del caso que les correspondió, elaborando una posible solución a través de un proyecto comunitario siguiendo las instrucciones de la Profesora que dictó el taller. Como cierre se organizaron los participantes en grupos por calle y cada grupo realizó un borrador de proyecto en función de una necesidad real de la comunidad. Comprometiéndose a darle una estructura formal según lo aprendido en el taller con el fin de exponerlo en el último encuentro dentro del intercambio de experiencias.

\subsubsection{Encuentro}

Taller II: Intercambio De Experiencias Comunitarias. Actividad de cierre.

Objetivo General: Compartir experiencias de los resultados del programa.

\section{Objetivos Específicos:}

- Generar un espacio para el intercambio de experiencias.

- Propiciar y motivar a la creación de proyectos vinculados a las necesidades de la comunidad.

- Promover el trabajo cooperativo y en equipo.

Descripción de la experiencia: Este tercer encuentro con la comunidad de Los Naranjos, estuvo compartida con la asistencia de habitantes de otras comunidades cercanas, los cuales asistieron motivados por la invitación difundida por medio de una radio local. Posterior a este inicio, el desarrollo de la actividad se centró en comenzar un intercambio de experiencias. Los participantes que asistieron al taller anterior tenían como 
meta recopilar datos en su comunidad que pudieran sostener la factibilidad de proyectos producidos en el taller. Al finalizar la primera ronda de experiencias, hubo un receso de quince minutos luego del cual los participantes se incorporaron al recinto destinado para el taller.

Durante la segunda fase de la jornada, los participantes procedieron a realizar críticas constructivas en función de los criterios dados por la facilitadora durante la sesión previa al encuentro. Finalmente, y luego de una retroalimentación los participantes se retiraron con propuestas corregidas y autoevaluadas con la intención de aplicarlas en sus comunidades. Sin duda, la participación activa de los miembros de la comunidad del Barrio Los Naranjos determinó el logro de los objetivos propuestos.

\section{Conclusiones}

En cuanto a los resultados de la aplicación del programa de Formación en Proyectos Comunitarios, se concluyó que el efecto causado por sí mismo no se limitó a la comunidad del Barrio Los Naranjos, a quienes fue dirigido originalmente. También impactó en los habitantes de comunidades adyacentes que asistieron a los encuentros. Éste fue el caso de la comunidad de aprendizaje de la Misión Rivas, quienes participaron con la puesta en ejecución de una plaza ecológica para la escuela de la comunidad donde reciben clases en las noches. Ellos aprendieron a realizar y ejecutar proyectos de interés a la comunidad de la cual se estaban sirviendo. Igualmente, la comunidad del Barrio Los Naranjos participó en el diseño de propuestas como asfaltado de las calles y arreglos de averías en el sistema de aguas servidas, entre otros. La comunidad referida, supo cuáles eran las instancias legales, así como las instituciones y los procedimientos a los cuales acudir para presentar de manera formal las solicitudes pertinentes ante las necesidades de interés comunitario. 


\subsection{De la experiencia en cuestión puede concluirse lo siguiente:}

- Las condiciones legales en Venezuela propician la existencia de comunidades organizadas a través de una participación ciudadana activa y protagónica.

- Las comunidades disponen de programas de formación comunitaria por parte del Estado venezolano, los cuales tienen como objetivo orientar en cuanto a la gestión y desarrollo de proyectos comunitarios.

- Existe el interés y la necesidad por parte de estas comunidades de conducir su propio desarrollo social.

- La comunidad de Los Naranjos cuenta con el conocimiento y las orientaciones necesarias obtenidas del Programa de Formación en Proyectos Comunitarios desarrollado en su comunidad, para elaborar y ejecutar proyectos que beneficien a su colectivo.

- Los proyectos comunitarios posibilitan la integración estimulando en los habitantes de la comunidad el sentido de pertenencia y el trabajo en equipo.

Asimismo, es oportuno destacar que las comunidades organizadas representan un factor determinante para el desarrollo social del Estado, que desde el contexto social en Venezuela demuestra un patrón característico de participación comunitaria, aunado a la tradición cultural muy propia de ciertos grupos sociales. Los cuales, independientemente de su origen y/o diversidad cultural, convergen en un fin común que es el bienestar social. Ante esta perspectiva, las comunidades organizadas han encontrado en el marco constitucional de las leyes el espacio propicio para el manejo de su participación activa y protagónica dentro de la transformación social y paulatina de las comunidades en Venezuela en los últimos años. 


\section{Referencias}

Brizuela, Z., Sáez, A., \& Jústiz, M. (2015). Metodología para la Educación Comunitaria. Humanidades Médicas, 15(1), 107-127, e-ISSN: 17278120. Recuperado de:

http://scielo.sld.cu/scielo.php?script=sci arttext\&pid=S1727$\underline{81202015000100007 \& \text { Ing }=e s \& t \operatorname{lng}=e s}$

Eizagirre, M., \& Zabala, N. (2006). Investigación-Acción Participativa (AIP). Diccionario de Acción Humanitaria y Cooperación al Desarrollo. España: Universidad del País Vasco; Instituto de Estudios sobre Desarrollo y Cooperación Internacional - hegoa. Recuperado de: http://www.dicc.hegoa.ehu.es/listar/mostrar/132

Ley Orgánica de los Consejos Comunales (2010a,b,c). Definiciones. Gaceta Oficial Nº 39.377 del 2 marzo. República Bolivariana de Venezuela: La Asamblea Nacional.

Ley Orgánica de Educación (2009a,b). La educación. Gaceta Oficial № 5.929 Extraordinario del 15 de agosto. República Bolivariana de Venezuela: La Asamblea Nacional. Recuperado de:

https://www.urbe.edu/portal-biblioteca/descargas/Ley-Organica\%20deEducacion.pdf

Maslow, A. (1991). Motivación y personalidad. Tercera edición, ISBN: 9788487189845. Madrid, España: Ediciones Díaz de Santos, S.A.

Mballa, L. (2017). Desarrollo local y microfinanzas como estrategias de atención a las necesidades sociales: un acercamiento teórico conceptual. Revista mexicana de ciencias políticas y sociales, 62(229), 101-127, e-ISSN: 0185-1918. Recuperado de:

http://www.scielo.org.mx/scielo.php?script=sci arttext\&pid=S0185$19182017000100101 \&$ lng=es\&tlng=es

Páez, A. (2017). Formación Docente en Técnicas de Superaprendizaje Aplicadas a La Enseñanza de la Matemática en la Educación 
Secundaria. Revista Scientific, 2(6), 10-28, e-ISSN: 2542-2987. Recuperado de:

https://doi.org/10.29394/scientific.issn.2542-2987.2017.2.6.1.10-28 Palomo, M. (2010). Liderazgo y motivación de equipos de trabajo. Sexta edición, ISBN: 978-84-7356-678-0. Madrid, España: ESIC Editorial. Requena, Y. (2018). Investigación Acción Participativa y Educación Ambiental. Revista Scientific, 3(7), 289-308, e-ISSN: 2542-2987. Recuperado de:

https://doi.org/10.29394/Scientific.issn.2542-2987.2018.3.7.15.289-308 UPEL (2016). Manual de Trabajos de Grado de Especialización y Maestría y Tesis Doctorales. 5ta Edición, ISBN: 980-273-441-1. Caracas, Venezuela: Fondo Editorial de la Universidad Pedagógica Experimental Libertador, FEDUPEL. Recuperado de:

https://es.slideshare.net/mirnalitaguirrez/manual-upel-2016-1pdf 


\section{Lisbeth María Cedeño de Veracierto \\ e-mail: lizbethcedeno@gmail.com}

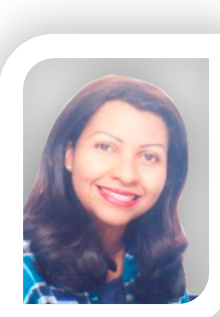

Nacida en Valencia, estado Carabobo, Venezuela, el 24 de noviembre de 1968. Egresada del Instituto Universitario Pedagógico Monseñor Rafael Arias Blanco (IUPMA) en Educación Comercial en el año 2007; Diplomado en Educación Superior en la Universidad Nacional Experimental Simón Rodríguez (UNESR) en el año 2009; Especialización en Educación para la Gestión Comunitaria en la Universidad Pedagógica Experimental Libertador (UPEL), Núcleo Valencia en el año 2011; Doctorado en Educación, desarrollo de tesis en la Universidad Pedagógica Experimental Libertador (UPEL), Maracay, Estado Aragua en el periodo 20152019; Ejercicio docente desde el año 1993 hasta la fecha; artes plásticas en educación escolar y de bachillerato internacional; metodología de la investigación y redacción de textos académicos a nivel universitario en el periodo 2015-2019.

El contenido de este manuscrito se difunde bajo una Licencia de Creative Commons ReconocimientoNoComercial-Compartirlgual 4.0 Internacional 